\title{
Editorial: Ecology, Evolution, and Behavior of Viviparous Fishes
}

\author{
J. Jaime Zúñiga-Vega ${ }^{1 *}$, Andrea S. Aspbury ${ }^{2}$, Jerald B. Johnson ${ }^{3}$ and Bart J. A. Pollux ${ }^{4}$ \\ ' Departamento de Ecología y Recursos Naturales, Facultad de Ciencias, Universidad Nacional Autónoma de Mexico, \\ Ciudad de Mexico, Mexico, ${ }^{2}$ Population and Conservation Biology Group, Department of Biology, Texas State University, \\ San Marcos, TX, United States, ${ }^{3}$ Evolutionary Ecology Laboratories, Department of Biology, Brigham Young University, \\ Provo, UT, United States, ${ }^{4}$ Experimental Zoology Group, Department of Animal Sciences, Wageningen University, \\ Wageningen, Netherlands
}

Keywords: behavioral traits, life-history evolution, livebearers, morphology, viviparity, Poeciliidae, predation, sexual selection

\section{Editorial on the Research Topic}

Ecology, Evolution, and Behavior of Viviparous Fishes

\section{INTRODUCTION}

Few organisms have contributed more to our understanding of ecology, evolution, and behavior than freshwater viviparous fishes (Meffe and Snelson, 1989; Uribe and Grier, 2005, 2010; Evans et al., 2011). These encompass freshwater species from several families, including Poeciliidae, Goodeidae, Anablepidae, and Zenarchopteridae. The poeciliids, a clade of almost 300 species (at last count), have been particularly important as research targets; and there is growing interest in other groups, including the zenarchopterids, the freshwater viviparous halfbeaks (Farhana et al., 2018). The former are primarily distributed throughout the Americas and the Caribbean, occurring in a variety of selective environments and ecological conditions, with the latter found in tropical Asia and New Guinea. Species in both groups tend to be integral to the ecological communities that they occupy. They are also strikingly important as models for understanding both microand macro-evolutionary processes. Indeed, they have proven to be critical to our understanding of pre- and post-copulatory sexual selection, complex social behavior, learning and cognition, the origin of unisexual species, genetic variation in natural populations, assembly of freshwater communities, and even the formation of tumors (Vrijenhoek, 1994; Evans and Pilastro, 2011; Krause et al., 2011; Rios-Cardenas and Morris, 2011; Lu et al., 2018). Further, their unique form of viviparous reproduction has made them the target of numerous studies focused on the anatomy and physiology related to internal fertilization and live birth (Uribe and Grier, 2005, 2010). At last count, almost 9,000 manuscripts have been published on just the poeciliid livebearing fishes.

One of the strengths of studying livebearing fishes is that they exhibit such a variety of phenotypic adaptations. This is true of evolutionary diversification among species, but is also prominently seen in evolutionary divergence within species. This is due in part to their ability to occupy such a wide range of habitats (Johnson and Bagley, 2011). Although most livebearing fishes are small stream specialists, they are known to tolerate extreme differences in water temperature, salinity, stream flow rate, water chemistry, and a variety of other abiotic factors (Torres-Dowdall et al., 2013; Martínez et al., 2016; Riesch et al., 2016). This range of tolerance makes them remarkably effective at invading new habitats, which has occurred historically with some species introduced globally to control mosquitos far outside their natural range, and some species introduced outside their ranges as a result of the aquarium trade (Maddern et al., 2011; Santi et al., 2020). 
With so much already known, it may seem that there is little still to learn from these fishes. Yet, in many ways, we are only now beginning to uncover the potential of these fishes to inform our understanding of several fundamental concepts in biology. Some of this potential is driven by technological advances that allow us to answer questions in new ways. For example, the ability to peer into the genomes of species, or to integrate long-term field studies with controlled laboratory experiments, offer the promise of new insights. Some potential lies in the fact that more work is being done on species that previously have received little or no attention, providing understanding that uniquely comes from comparative work (e.g., Furness et al., 2019, 2021). And some potential lies in knowing some systems so well that we can now begin to integrate knowledge across disciplines that previously were considered independently. Here, we introduce a set of papers published in a Research Topic of Frontiers in Ecology and Evolution focused on recent work in viviparous fishes. These articles can be organized into five broad categories: Behavior, Ecology, Life-History Evolution, Morphology, and Sexual Selection. Some articles used invasive species as model systems and, thus, our Research Topic also contributes to our understanding of how viviparity may be related to the invasive potential of some fish species. Further, we provide a general overview of the significant contributions contained in each article and suggest questions for future investigations.

\section{BEHAVIOR}

Several articles explore behavior in relation to environmental change. For example, Lukas, Kalinkat et al. examined behavior and invasion success by testing whether dispersing individual guppies (Poecilia reticulata) exhibit behavioral types that differ from those remaining in the source population. They found that guppies exhibit a stable behavioral syndrome composed of boldness and activity, even in the presence of strong seasonal changes in abiotic environmental factors, indicating that individuals with high activity levels are also bolder. Their work showed that when individuals occupy unpredictable and fluctuating environments, having consistent behavioral expressions could help guppies successfully invade, and adapt to disturbed habitats. Santiago-Arellano et al. also examined behavior in invasive guppies asking if heterospecifics (both native and other invasive viviparous fish) affect guppies' refuge emergence latency. When guppies were with other guppies, other invaders, and native goodeids, they had a shorter emergence latency than when alone. Latency reduction was highest when with conspecifics or the invader Poeciliopsis gracilis. Their work shows that established invaders might provide benefits to new invaders.

In an invasive livebearing poeciliid, Gambusia affinis, Miner et al. investigated if they were behaviorally or physiologically tolerant to the environmental perturbation of artificial light at night (ALAN). They found that exposure to ALAN lowered glucose levels in the brain and decreased swimming activity, but did not significantly affect cortisol release rates, reproduction, survival, or growth. They suggest that G. affinis may behaviorally adjust to ALAN rather than modulate their endocrine stress response, which might be one mechanism of their tolerance to disturbed habitats. Devigili et al. found that variation in canopy cover and water flow influenced sexual interactions and locomotor behaviors in the pygmy halfbeak Dermogenys collettei (family Zenarchopteridae). Their work supports the argument that gathering basic ecological and behavioral information from wild populations can lead to a better understanding of the interaction between natural and sexual selection in viviparous fishes.

Two studies addressed how lateralization or handedness could affect behaviors. Johnson et al. showed that variation in male Xenophallus umbratilis gonopodial corkscrew morphology (either left- or right-handed) was linked to decision making behaviors, including behaviors not associated with mating. Interestingly, right and left morph males responded with opposite directional behaviors when presented with the same stimuli. Connelly and Ryan compared visual lateralization in the asexual, gynogenetic Amazon molly (Poecilia formosa), and the sexually reproducing, and sexually parasitized Sailfin molly $(P$. latipinna). Because Amazon mollies are hybrids with P. latipinna as one parental species, these two species may have similar lateralization. Alternatively, variability or plasticity in this trait might aid in the evolutionary maintenance of the asexual species. In their experiments they failed to detect a significant eye bias in either species, and the degree of variation in visual lateralization was similar between the asexual and sexual species.

\section{ECOLOGY}

Lukas, Auer et al. examined how changes in dissolved oxygen that occur throughout the day in a sulfidic spring affect predatorprey interactions. They documented that as dissolved oxygen decreases from morning to afternoon, Poecilia sulphuraria increase their rates of aquatic surface respiration, making themselves more vulnerable to bird predators. This interesting study demonstrates that abiotic factors that affect prey species may have strong indirect effects on their predators. Langerhans et al. conducted a thorough study of the consumption of hard-shelled prey (durophagy), which are difficult to digest and hence are a costly type of prey. They found that durophagy is quite common among teleost fishes and is widespread across populations of the viviparous fish Gambusia holbrooki. According to their findings, durophagy (in particular molluscivory) is common under conditions of food limitation. This study greatly contributes to our understanding of the benefits of consuming costly prey, the ecological conditions that elicit its occurrence, and the mechanisms by which predators alleviate its costs.

\section{LIFE-HISTORY EVOLUTION}

By focusing on an adaptive radiation of Gambusia hubbsi inhabiting blue holes in Bahamas, Hulthén et al. disentangled the effects of predation and food availability on the evolution of life-history traits. They observed a complex interaction between 
these two selective factors shaping fecundity, offspring size, body growth, and age at maturity. One of the greatest strengths of this study is that it dissociates the effects of predation and food availability, which usually covary across natural populations. In contrast, Golden et al. also examined effects of predation intensity on life history diversification in Alfaro cultratus and found no differences in size at maturity, fecundity or offspring size between low- and high-predation environments. Apparently, morphological restrictions limit the selective effects of predation because females from this species have a narrow body and do not develop a distended abdomen during pregnancy. This study thus stands out because it offers a different perspective on the potential effects of predators on life-history evolution, which may not always be as strong as observed in other viviparous species.

A simultaneous examination of multiple selective agents potentially causing intraspecific life-history variation in Poeciliopsis prolifica was conducted by Roth-Monzón et al. Their results revealed that both population density and interspecific competition are strong selective agents for most life-history traits. This important contribution demonstrates that other ecological factors, in addition to the well-known effect of predation, interact in shaping life-history diversification. Saleh-Subaie et al. examined the Trexler-DeAngelis model (Trexler and DeAngelis, 2003, 2010), which is one of the most prominent hypotheses that attempts to explain the causes and consequences of the evolution of matrotrophy (post-fertilization embryonic nutrition) from the ancestral lecithotrophy (pre-fertilization embryonic nutrition). They observed in five species of the genus Poeciliopsis that females with greater degrees of matrotrophy had poorer body condition, which is consistent with one of the predicted consequences of matrotrophy according to the TrexlerDeAngelis model. This finding reveals that post-fertilization embryonic nutrition entails severe energetic costs for females of viviparous fishes.

\section{MORPHOLOGY}

Viviparous fishes are excellent models for examining questions about the origin of, and importance of morphological variation. Safian et al. examined how poeciliids could be used as model systems to test hypotheses about fish fin regeneration. They reviewed the current knowledge about the mechanisms behind fin regeneration in fish and noted that the Poeciliidae are a model family for comparative studies of fin regeneration because these fishes live in very different habitat types, have a well-defined phylogeny, and exhibit an incredible amount of variation in morphology and reproductive traits. Langerhans and Rosa-Molinar extended this perspective by investigating the basis of morphological innovation in the gonopodial structure of Gambusia fishes. They document changes in the ano-urogenital vertebral region that have facilitated divergence in swimming abilities and body shape between habitats that vary in predation regimes. This study suggests that the structure of this body region, that originally evolved in the sperm transfer context, has been co-opted to alter swimming performance, and therefore whole-organism survival, facilitating phenotypic diversification when predation regimes vary. Zerulla and Stoddard reviewed the general biology of the polymorphic melanic side-spotting patterns formed by macromelanophores, that are present in some species of Gambusia, Limia, Phalloceros, Poecilia, and Xiphophorus. The side spot patterns formed by macromelanophores are influenced by the presence of dominant genes, acting in a polygenic fashion, that can be either sex-linked or autosomal. Despite the influence of dominant genes, there is often a low frequency of the side-spotting morph in populations, suggesting that there must be selective pressures influencing these traits. However, while side-spotting morphology had wide variation across taxa they did not find that it correlates with sexual selection.

\section{SEXUAL SELECTION}

Much of our knowledge of patterns and processes of sexual selection comes from studies of viviparous fishes (Basolo, 1995; Evans and Pilastro, 2011; Rios-Cardenas and Morris, 2011). Several articles in our Research Topic add to this knowledge. Although theory predicts strong species recognition as a first step in mate choice, Magellan and García-Berthou discuss how selection for accurate species recognition may not be as strong in invasive species with coercive mating systems, such as mosquitofish (Gambusia holbrooki). The pairing of rapid learning of species identity with an innate predisposition for sex recognition over species recognition, could be a factor in the invasive success of mosquitofish. Dekker et al. examined multiple paternity in Poecilia gillii and found that over $70 \%$ of reproductive females carry embryos from different fathers. They documented that some broods were sired by up to nine different males and in most cases these males did not contribute with a similar number of offspring, thus revealing interesting reproductive skew. This is a noteworthy contribution to our knowledge of the processes of post-copulatory sexual selection that occur in viviparous fishes. Reznick et al. summarized the consequences that the evolution of matrotrophy from the ancestral lecithotrophy has brought on the evolution of male traits associated with sexual selection. They emphasize that males from species with matrotrophy and placentas are less likely to have elaborate ornaments and courtship behavior, are smaller relative to females, and have longer gonopodia. This association indicates that the evolution of matrotrophy led to a shift from pre-copulatory to post-copulatory sexual selection. They also document an accelerated speciation rate in species that exhibit clear signs of pre-copulatory mate choice.

\section{FUTURE DIRECTIONS}

\section{Convergent Evolution}

Convergent evolution of similar phenotypes in distinct evolutionary lineages is an important phenomenon that has shaped the natural history on earth. Viviparous fish families such as the Poeciliidae, Anablepidae, Goodeidae (all three in Order Cyprinodontiformes), and Zenarchopteridae (Order Beloniformes) are excellent systems to study convergent evolution. They form groups of closely related species that occur 
in different habitats characterized by a tremendous variation in biotic and abiotic conditions. This has produced a stunning diversity in morphology, physiology, behavior, life-history, and reproductive adaptations. Many of these features evolved repeatedly across the family tree and vary both within and among species. Family-wide phylogenetic comparative approaches that assess how phenotypic features co-evolve across species, or evolve in association with ecological factors, will allow us to address important questions. For example, is evolution repeatable within and among families? Is phenotypic convergence the result of common solutions to similar selective pressures? To what extent does convergent evolution produce similar (but not necessarily always identical) phenotypes? Finally, and equally interesting, do similar selective pressures always lead to similar convergent adaptations or can they also lead to different (non-convergent) phenotypic adaptations? Answers to these captivating questions from live-bearing fishes may significantly further our general understanding of the evolution of phenotypic convergence at both micro- and macro-evolutionary levels (Losos, 2011; Wake et al., 2011).

\section{Genomics of Adaptation}

Another promising avenue of future research is one that focusses on uncovering the molecular pathways underlying the convergent evolution of complex adaptations. Recent advances in sequencing technologies, declines in sequencing costs, and innovative computational approaches mark the beginning of a genomics revolution (Giani et al., 2020), with more and more fish genomes becoming rapidly available. The field of comparative genomics is bringing this full genome information together with research on ecological selective factors and phenotypic adaptations, giving rise to new opportunities for studying the genomic basis of complex adaptations. We foresee that future studies will focus on two timely topics. The first centers on uncovering the genomic basis of complex trait evolution. The genetic possibilities are countless and may include changes in protein coding genes, gene duplications/losses, or changes in non-coding regulatory elements that result in adjustments of the spatiotemporal expression patterns of key genes. Moreover, these changes may range from relatively minor (epi)genetic changes in only a few, or even single, key genes, to more elaborate modifications at multiple locations in different developmental pathways (Wagner and Altenberg, 1996; Carroll, 2008). The second topic focuses on the extent to which convergent evolution in independent lineages is associated with parallel or alternative changes at the genomic level. Comparing the genomes of populations or species that independently evolved new phenotypic traits allows the identification of genomic differences associated with these phenotypes (Elmer and Meyer, 2011; Stern, 2013; van Kruistum et al., 2021). It is noteworthy to point out that such comparative approaches become statistically more powerful in identifying phenotype-related genes as the number of independent origins included in the study increases.

\section{Population Dynamics and Conservation}

Another important aspect that deserves attention is the population status of most species of viviparous fishes. To date, we know little about their demographic trends or conservation needs. A few studies have estimated sex- or stage-specific mortality rates, but the main purpose of these studies has been to identify the sources of extrinsic mortality and its microevolutionary consequences, rather than to know if populations are growing, stable, or declining (Reznick and Bryant, 2007; Johnson and Zúñiga-Vega, 2009; Zúñiga-Vega et al., 2012). In the light of the current global situation, with numerous water bodies drying up or increasing in pollution (Kingsford, 2011; Wen et al., 2017), we must make additional efforts to estimate demographic parameters such as mortality, recruitment, and population growth rates. Furthermore, temperature is increasing in many water bodies, mainly near human settlements, a phenomenon called "urban hot-tubs" (Brans et al., 2018). We still do not understand how such warmer temperatures are affecting the populations of aquatic organisms.

Certainly, many species of viviparous fishes are widespread and others (e.g., some poeciliid species) are considered invasive species, with numerous populations growing in areas outside their native geographic ranges (Albornoz-Garzón and VillaNavarro, 2017; Santi et al., 2020). However, many other species have restricted distributions (e.g., most species in the family Goodeidae) and inhabit regions with high rates of habitat degradation (Meffe et al., 1983; Domínguez-Domínguez et al., 2006). Some studies have made significant efforts to propose conservation status of particular species, aiming to call the attention of managers and government environmental agencies (Tobler and Plath, 2009; Tobler and Schlupp, 2009; Echelle et al., 2013). Still, demographic characteristics of most viviparous species remain entirely unknown and quantitative information is urgently needed to guide future conservation strategies.

\section{CONCLUDING REMARKS}

The breadth of research on livebearing fishes-that which has been completed, is underway, or is being planned-is truly astounding. The articles contained in this Research Topic of Frontiers in Ecology and Evolution capture some of this breadth with clever studies that expand the boundaries of our understanding. But they also reveal biases in this larger body of work. Some species are much more extensively studied than others; some biological disciplines are over-represented; and some important questions have yet to be asked. Perhaps the most exciting thing that will come from work on livebearing fishes is the synthesis of biological knowledge that can occur. Rather than viewing organisms as a collection of traits independently evolving, several species of livebearing fishes are poised to be understood as an integrated expression of genes interacting with a complex environment-this approach offers a way to more fully understand the fundamental bauplan of fishes, and maybe of all vertebrates (Gould, 2002). Similarly, as we come to examine more and more species of livebearing fishes, our understanding of the contraints and flexibility of evolution will also become clearer. Examples of parallel evolution already excite the possibility of understanding the predictability of evolutionary change (Reznick et al., 1996; Johnson, 2001; Ingley et al., 2014; Tobler et al., 2015). And of course, the applied use of this group of fishes continues 
to grow, including their use to understand disease, development, and conservation, to name but a few. Clearly, future work studying freshwater viviparous fishes holds much promise.

\section{AUTHOR CONTRIBUTIONS}

All authors contributed equally to this Editorial article and to the organization of the Research Topic.

\section{REFERENCES}

Albornoz-Garzón, J. G., and Villa-Navarro, F. A. (2017). Range extension of the invasive fish Xiphophorus maculatus (Günther, 1866) (Cyprinodontiformes: Poeciliidae) in the upper Magdalena river basin, Colombia. Check List 13:2149. doi: $10.15560 / 13.3 .2149$

Basolo, A. L. (1995). Phylogenetic evidence for the role of a pre-existing bias in sexual selection. Proc. $R$ Soc. B Biol. Sci. 259, 307-311. doi: 10.1098/rspb.1995.0045

Brans, K. I., Engelen, J. M. T., Souffreau, C., and De Meester, L. (2018). Urban hot-tubs: local urbanization has profound effects on average and extreme temperatures in ponds. Landsc. Urban Plan. 176, 22-29. doi: 10.1016/j.landurbplan.2018.03.013

Carroll, S. B. (2008). Evo-devo and an expanding evolutionary synthesis: a genetic theory of morphological evolution. Cell 134, 25-36. doi: 10.1016/j.cell.2008.06.030

Domínguez-Domínguez, O., Martínez-Meyer, E., Zambrano, L., and Pérez-Ponce de León, G. (2006). Using ecological-niche modeling as a conservation tool for freshwater species: live-bearing fishes in central Mexico. Conserv. Biol. 20, 1730-1739. doi: 10.1111/j.1523-1739.2006.00588.x

Echelle, A. A., Lozano Vilano, M. L., Baker, S., Wilson, W. D., Echelle, A. F., Garrett, G. P., et al. (2013). Conservation genetics of Gambusia krumholzi (Teleostei: Poeciliidae) with assessment of the species status of G. clarkhubbsi and hybridization with G. speciosa. Copeia 2013, 72-79. doi: 10.1643/CG-11-167

Elmer, K. R., and Meyer, A. (2011). Adaptation in the age of ecological genomics: insights from parallelism and convergence. Trends Ecol. Evol. 26, 298-306. doi: $10.1016 /$ j.tree.2011.02.008

Evans, J. P., and Pilastro, A. (2011). "Postcopulatory sexual selection," in Ecology and Evolution of Poeciliid Fishes, eds J. P. Evans, A. Pilastro, and I. Schlupp (Chicago, IL: The University of Chicago Press), 197-208. doi: 10.7208/chicago/9780226222769.001.0001

Evans, J. P., Pilastro, A., and Schlupp, I. (2011). Ecology and Evolution of Poeciliid Fishes. Chicago, IL: The University of Chicago Press.

Farhana, S. N., Muchlisin, Z. A., Duong, T. Y., Tanyaros, S., Page, L. M., Zhao, Y., et al. (2018). Exploring hidden diversity in Southeast Asia’s Dermogenys spp. (Beloniformes: Zenarchopteridae) through DNA barcoding. Sci. Rep. 8:10787. doi: 10.1038/s41598-018-29049-7

Furness, A. I., Avise, J. C., Pollux, B. J. A., Reynoso, Y., and Reznick, D. N. (2021). The evolution of the placenta in poeciliid fishes. Curr. Biol. 31, 2004-2011. doi: 10.1016/j.cub.2021.02.008

Furness, A. I., Pollux, B. J. A., Meredith, R. W., Springer, M. S., and Reznick, D. N. (2019). How conflict shapes evolution in poeciliid fishes. Nat. Commun. 10:3335. doi: 10.1038/s41467-019-11 307-5

Giani, A. M., Gallo, G. R., Gianfranceschi, L., and Formenti, G. (2020), Long walk to genomics: history and current approaches to genome sequencing and assembly. Comput. Struct. Biotechnol. J. 18, 9-19. doi: $10.1016 /$ j.csbj.2019.11.002

Gould, S. J. (2002). The Structure of Evolutionary Theory. Cambridge, MA: Harvard University Press. doi: 10.2307/j.ctvjsf433

Ingley, S. J., Billman, E. J., Belk, M. C., and Johnson, J. B. (2014). Morphological divergence driven by predation environment within and between species of Brachyrhaphis fishes. PLoS ONE 9:e90274. doi: 10.1371/journal.pone.0090274

Johnson, J. B. (2001). Adaptive life-history evolution in the livebearing fish Brachyrhaphis rhabdophora: genetic basis for parallel divergence in age and size

\section{ACKNOWLEDGMENTS}

We are very grateful to all the contributing authors for sharing with us their research findings. Their manuscripts have made the Research Topic on viviparous fishes a great success. We also thank all the referees, acknowledged on the first page of each article, for providing constructive and thoughtful comments on manuscript drafts.

at maturity and a test of predator-induced plasticity. Evolution 55, 1486-1491. doi: 10.1111/j.0014-3820.2001.tb00668.x

Johnson, J. B., and Bagley, J. C. (2011). "Ecological drivers of life-history divergence," in Ecology and Evolution of Poeciliid Fishes, eds. J. P. Evans, A. Pilastro, and I. Schlupp (Chicago, IL: The University of Chicago Press), 38-49.

Johnson, J. B., and Zúñiga-Vega, J. J. (2009). Differential mortality drives life-history evolution and population dynamics in the fish Brachyrhaphis rhabdophora. Ecology 90, 2243-2252. doi: 10.1890/07-1672.1

Kingsford, R. T. (2011). Conservation management of rivers and wetlands under climate change - a synthesis. Mar. Freshw. Res. 62, 217-222. doi: 10.1071/MF11029

Krause, J., James, R., and Croft, D. P. (2011). "Group living," in Ecology and Evolution of Poeciliid Fishes, eds J. P. Evans, A. Pilastro, and I. Schlupp (Chicago, IL: The University of Chicago Press), 145-154.

Losos, J. B. (2011). Convergence, adaptation, and constraint. Evolution 65, 1827-1840. doi: 10.1111/j.1558-5646.2011.01289.x

Lu, Y., Boswell, M., Boswell, W., Kneitz, S., Hausmann, M., Klotz, B., et al. (2018). Comparison of Xiphophorus and human melanoma transcriptomes reveals conserved pathway interactions. Pigment Cell Melanoma Res. 31, 496-508. doi: $10.1111 /$ pcmr.12686

Maddern, M. G., Gill, H. S., and Morgan, D. L. (2011). Biology and invasive potential of the introduced swordtail Xiphophorus hellerii Heckel (Poeciliidae) in Western Australia. Aquat. Conserv. Mar. Freshw. Ecosyst. 21, 282-291. doi: $10.1002 /$ aqc.1186

Martínez, J. D., Cadena, C. D., and Torres, M. (2016). Critical thermal limits of Poecilia caucana (Steindachner, 1880) (Cyprinodontiformes: Poeciliidae). Neotrop. Ichthyol. 14:e150171. doi: 10.1590/1982-0224-20150171

Meffe, G. K., Hendrickson, D. A., Minckley, W. L., and Rinne, J. N. (1983). Factors resulting in decline of the endangered Sonoran topminnow Poeciliopsis occidentalis (Atheriniformes: Poeciliidae) in the United States. Biol. Conserv. 25, 135-159. doi: 10.1016/0006-3207(83)90057-5

Meffe, G. K., and Snelson, F. F. Jr., (eds.). (1989). Ecology and Evolution of Livebearing Fishes (Poeciliidae). Englewood Cliffs, NJ: Prentice Hall.

Reznick, D., and Bryant, M. (2007). Comparative long-term mark-recapture studies of guppies (Poecilia reticulata): differences among high and low predation localities in growth and survival. Ann. Zool. Fennici 44, 152-160.

Reznick, D. N., Rodd, F. H., and Cardenas, M. (1996). Life-history evolution in guppies (Poecilia reticulata: Poeciliidae). IV. Parallelism in life-history phenotypes. Am. Nat. 147, 319-338. doi: 10.1086/285854

Riesch, R., Tobler, M., Lerp, H., Jourdan, J., Doumas, T., Nosil, P., et al. (2016). Extremophile Poeciliidae: multivariate insights into the complexity of speciation along replicated ecological gradients. BMC Evol. Biol. 16:136. doi: 10.1186/s12862-016-0705-1

Rios-Cardenas, O., and Morris, M. R. (2011). "Precopulatory sexual selection," in Ecology and Evolution of Poeciliid Fishes, eds. J. P. Evans, A. Pilastro, and I. Schlupp (Chicago, IL: The University of Chicago Press), 187-196.

Santi, F., Riesch, R., Baier, J., Grote, M., Hornung, S., Jüngling, H., et al. (2020). A century later: adaptive plasticity and rapid evolution contribute to geographic variation in invasive mosquitofish. Sci. Tot. Environ. 726:137908. doi: $10.1016 /$ j.scitotenv.2020.137908

Stern, D. L. (2013). The genetic causes of convergent evolution. Nat. Rev. Genet. 14, 751-764. doi: 10.1038/nrg3483

Tobler, M., and Plath, M. (2009). Threatened fishes of the world: Poecilia sulphuraria (Alvarez, 1948) (Poeciliidae). Environ. Biol. Fishes 85, 333-334. doi: 10.1007/s10641-009-9506-3 
Tobler, M., Scharnweber, K., Greenway, R., Passow, C. N., Arias-Rodriguez, L., and García-De-León, F. J. (2015). Convergent changes in the trophic ecology of extremophile fish along replicated environmental gradients. Freshw. Biol. 60, 768-780. doi: 10.1111/fwb.12530

Tobler, M., and Schlupp, I. (2009). Threatened fishes of the world: Poecilia latipunctata Meek, 1904 (Poeciliidae). Environ. Biol. Fishes 85, 31-32. doi: 10.1007/s10641-009-9451-1

Torres-Dowdall, J., Dargent, F., Handelsman, C. A., Ramnarine, I. W., and Ghalambor, C. K. (2013). Ecological correlates of the distribution limits of two poeciliid species along a salinity gradient. Biol. J. Linn. Soc. 108, 790-805. doi: 10.1111/bij.12031

Trexler, J. C., and DeAngelis, D. L. (2003). Resource allocation in offspring provisioning: an evaluation of the conditions favoring the evolution of matrotrophy. Am. Nat. 162, 574-585. doi: 10.1086/378822

Trexler, J. C., and DeAngelis, D. L. (2010). "Modeling the evolution of complex reproductive adaptations in poeciliid fishes: matrotrophy and superfetation," in Viviparous Fishes II, eds M. C. Uribe and H. J. Grier (Homestead, FL: New Life Publications), 231-240.

Uribe, M. C., and Grier, H. J. (2005). Viviparous Fishes. Homestead, FL: New Life Publications.

Uribe, M. C., and Grier, H. J. (2010). Viviparous Fishes II. Homestead, FL: New Life Publications.

van Kruistum, H., Nijland, R., Reznick, D. N., Groenen, M. A. M., Megens, H.-J., and Pollux, B. J. A. (2021). Parallel genomic changes drive repeated evolution of placentas in live-bearing fish. Mol. Biol. Evol. 38, 2627-2638. doi: $10.1093 / \mathrm{molbev} / \mathrm{msab} 057$

Vrijenhoek, R. C. (1994). Unisexual fish: model systems for studying ecology and evolution. Annu. Rev. Ecol. Syst. 25, 71-96. doi: 10.1146/annurev.es.25.110194.000443

Wagner, G. P., and Altenberg, L. (1996). Complex adaptations and the evolution of evolvability. Evolution 50, 967-976. doi: 10.1111/j.1558-5646.1996.tb02339.x
Wake, D. B., Wake, M. H., and Specht, C. D. (2011). Homoplasy: from detecting pattern to determining process and mechanism of evolution. Science 331, 1032-1035. doi: 10.1126/science.11 88545

Wen, Y., Schoups, G., and van de Giesen, N. (2017). Organic pollution of rivers: combined threats of urbanization, livestock farming and global climate change. Sci. Rep. 7:43289. doi: 10.1038/srep4 3289

Zúñiga-Vega, J. J., Rodríguez-Reyes, F. R., Olivera-Tlahuel, C., MacedaCruz, R. J., Molina-Zuluaga, C. M., and Johnson, J. B. (2012) Intraspecific variation in mortality rates of the livebearing fish Poeciliopsis baenschi. Environ. Biol. Fishes 95, 259-273. doi: 10.1007/s10641-012-9 989-1

Conflict of Interest: The authors declare that the research was conducted in the absence of any commercial or financial relationships that could be construed as a potential conflict of interest.

Publisher's Note: All claims expressed in this article are solely those of the authors and do not necessarily represent those of their affiliated organizations, or those of the publisher, the editors and the reviewers. Any product that may be evaluated in this article, or claim that may be made by its manufacturer, is not guaranteed or endorsed by the publisher.

Copyright (c) 2022 Zúñiga-Vega, Aspbury, Johnson and Pollux. This is an open-access article distributed under the terms of the Creative Commons Attribution License (CC $B Y)$. The use, distribution or reproduction in other forums is permitted, provided the original author(s) and the copyright owner(s) are credited and that the original publication in this journal is cited, in accordance with accepted academic practice. No use, distribution or reproduction is permitted which does not comply with these terms. 